\title{
Determinação de parâmetros de reservatório através do efeito de polarização induzida observado em medidas elétricas espectrais de laboratório
}

\author{
Marinho, L. P.; Dias, C. A.; Santos, V. H.; LENEP-UENF
}

Copyright 2018, SBGf - Sociedade Brasileira de Geofísica

Este texto foi preparado para a apresentação no VIII Simpósio Brasileiro de Geofísica, Salinópolis, 18 a 20 de setembro de 2018. Seu conteúdo foi revisado pelo Comitê Técnico do VIII SimBGf, mas não necessariamente representa a opinião da SBGf ou de seus associados. É proibida a reprodução total ou parcial deste material para propósitos comerciais sem prévia autorização da SBGf.

\section{Resumo}

A determinação de parâmetros de reservatório é de crucial importância para caracterização do fluxo de fluidos no interior dos poros nas rochas. Atualmente, muitas pesquisas têm voltado sua atenção para a relação entre parâmetros petrofísicos de reservatório e medidas elétricas do efeito de polarização induzida (Efeito IP). Com o objetivo de estender a compreensão sobre a relação do efeito IP e parâmetros de reservatório, foram realizadas medidas em laboratório com plugues de rocha da bacia de Campos, Brasil. Sua resistividade complexa espectral foi medida no intervalo de frequência $10^{-3} \mathrm{~Hz}$ a $10^{5} \mathrm{~Hz}$, representada em termos de fase e amplitude. Para a descrição analítica do efeito IP registrado, dois modelos elétricos foram empregados para ajustar os dados experimentais: o modelo de Dias e um modelo composto. A aplicação do modelo composto foi eficiente para o ajuste das discrepâncias entre a modelagem promovida pelo modelo de Dias e os dados experimentais no intervalo de frequências intermediárias, onde se acredita que as heterogeneidades do sistema rocha-fluido exercem forte influência. Os parâmetros do modelo de Dias fornecidos pelos dois modelos foram utilizados para estimar os valores de permeabilidade e raio médio de poro, com sucesso em ambos os casos. Desta análise, houve ainda a determinação do intervalo de frequências em que as medidas de resistividade complexa devem ser tomadas, para uma possível determinação da permeabilidade in situ.

\section{Introdução}

A polarização elétrica induzida (ou efeito IP) corresponde a um fenômeno de polarização de interface nas rochas, sendo a polarização por partículas de argilominerais disseminadas nos poros dessas rochas (polarização de membrana) a mais frequente em reservatórios de hidrocarbonetos. Com isso, é crescente o interesse da indústria de exploração e produção de petróleo em pesquisas que correlacionem as informações obtidas do efeito de IP com propriedades de rochas de reservatório, visando auxiliar na caracterização de zonas produtoras (Dias, 2000; Tong et al., 2006; Revil \& Florsch, 2010; Revil et al., 2012; Barreto \& Dias, 2014).

Historicamente, muitos modelos foram sugeridos para descrever o fenômeno de IP. Como mostrado e analisado por Dias (2000), conclui-se que apenas dois modelos apresentam a capacidade necessária para ajustar os dados experimentais de resistividade elétrica disponíveis em todo o espectro de frequências que abrange o efeito IP: o modelo de Dias (Dias, 1968, 1972, 2000) e o modelo Multi Cole-Cole (Pelton et al., 1978). Estes dois modelos são capazes de gerar até dois máximos de fase da resistividade elétrica. No entanto, o modelo proposto por Dias (2000), através da sua função constitutiva do meio, denominada condutividade de corrente total, tem a vantagem de descrever o fenômeno de polarização observado de forma analítica e com significado físico para todos os seus parâmetros. Já a função original de Cole-Cole, de natureza empírica, produz um único máximo de fase, restringindo o ajuste a somente uma parte das curvas complexas de resistividade espectral e sem uma explicação física consistente para todos os seus parâmetros.

Em Barreto \& Dias (2014), foi apresentado um esclarecimento significativo sobre os máximos de fase do espectro de resistividade elétrica. Neste trabalho, houve a demonstração de que o primeiro máximo de fase, às frequências mais baixas, está relacionado ao processo de difusão de cátions em solução, próximo à interface argilomineral-eletrólito dentro de uma estrutura elétrica denominada dupla camada elétrica de Helmholtz. Já o segundo máximo de fase, às altas frequências, está relacionado a um efeito resistivo-capacitivo devido à dupla camada elétrica de Helmholtz como um todo. Através da determinação do tempo de relaxação associado ao efeito de difusão, estes autores mostraram também ser possível quantificar a capacidade de escoamento de fluido através da rocha, com a determinação do parâmetro permeabilidade.

Com o objetivo de dar prosseguimento ao estudo da relação entre os parâmetros do modelo de Dias e propriedades de reservatório, foram obtidas em laboratório medidas de resistividade elétrica espectral de amostras de rocha de um campo produtor da Bacia de Campos. A partir da modelagem desses dados e dos parâmetros obtidos do modelo de Dias, foi possível obter valores para a permeabilidade para cada amostra. Além disso, seguindo teoria apresenta em Marinho (2018), foi possível determinar das curvas de resistividade, também às baixas frequências, estimativas do raio médio de poro efetivo do sistema poroso dessas amostras de rocha.

\section{Metodologia}

As amostras de rocha disponibilizadas pela ANP/Petrobras foram provenientes de um campo produtor da Bacia de Campos, consistindo de 8 plugues entre 1" e 11/2" de diâmetro, com identificação da orientação do corte do plugue no testemunho em relação ao eixo do poço $(\mathrm{H}=$ horizontal e $\mathrm{V}=$ vertical $)$ da zona de 
reservatório testemunhada. O procedimento experimental com essas amostras foi conduzido no Laboratório de Petrofísica do LENEP-UENF, em Macaé-RJ, consistindo das seguintes atividades:

Caracterização Geológica: obtenção de dados sobre a composição mineral (\%) das pastilhas de rocha pulverizadas (tamanho mesh $=270$ ) por difração e fluorescência de raios- $X$, através do método de Rietveld (1969), de forma a identificar os causadores do efeito IP.

Petrofísica Básica: A determinação da porosidade $\phi \quad(\%)$ em laboratório seguiu as técnicas descritas em API (1998), utilizando gás He e o método conhecido como "Boyle's Law Double Cell". Já a permeabilidade absoluta $k$ (mD) das amostras de rocha foi realizada pela injeção de gás $\mathrm{N}_{2}$ de forma uniaxial através do plugue, inserido em um coreholder do tipo Hassler com pressão de confinamento de 500psi. Todos os ensaios com as amostras ocorreram à temperatura constante $\left(22,0 \pm 1,0^{\circ} \mathrm{C}\right)$ e sob um regime steady-state. A partir deste conjunto de dados, foi determinado o valor de $k$ em cada plugue através da Lei de Darcy (Bear, 1972).

Petrofísica Elétrica e Modelagem dos Dados Experimentais: Primeiramente, as amostras de rocha foram saturadas por um eletrólito de $\mathrm{NaCl}$ de baixa concentração $\left(10^{-2} \mathrm{M}\right)$, preparado com água ultrapura ( $\rho=18,2 \mathrm{M} \Omega . \mathrm{cm} @ 25^{\circ} \mathrm{C}$ ) e deionizada. A saturação da rocha pelo eletrólito foi obtida seguindo a metodologia apresentada em Maerefat et al. (1990). Posteriormente, o plugue saturado foi inserido em um porta amostras composto por 4 eletrodos de prata recobertos por ouro (ver Figura 1), não polarizáveis, e que não reagem quimicamente com a solução de $\mathrm{NaCl}$. O porta amostras citado é acoplado ao equipamento 1260 Impedance Analyser, onde foi registrada a variação da impedância elétrica complexa no espectro de frequências de $10^{-3} \mathrm{~Hz}$ a $10^{5} \mathrm{~Hz}$, à temperatura constante e idêntica às registradas durante os ensaios de porosidade e permeabilidade.

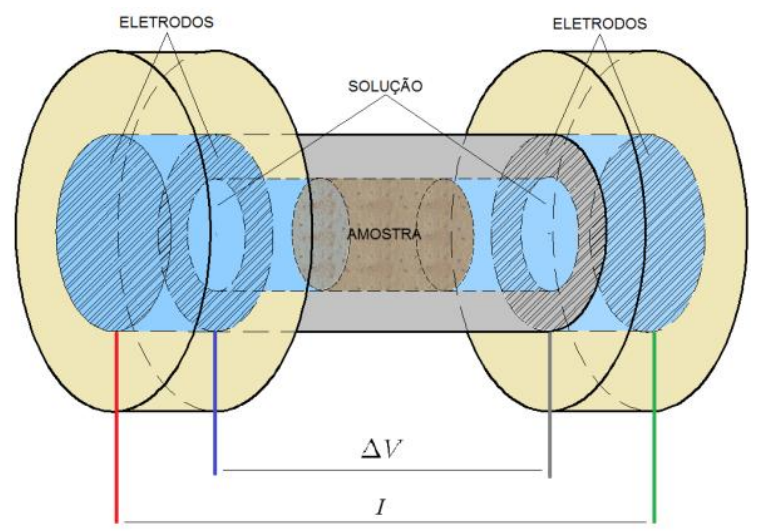

Figura 1 - Visão interna do porta amostras para determinação da impedância elétrica, destacando a disposição do plugue de rocha e dos pares de eletrodos em seu interior (discos hachurados).
A resistividade elétrica complexa pode ser obtida da impedância elétrica complexa através da relação:

$$
\rho^{*}=\frac{Z^{*}}{g}=\rho^{\prime}-i \rho^{\prime \prime},
$$

onde $\rho^{*}$ é a resistividade elétrica complexa, $Z^{*}$ é a impedância elétrica complexa, $g$ é o fator geométrico da amostra, $i=\sqrt{-1}$ é a unidade complexa e $\rho^{\prime}$ e $\rho^{\prime \prime}$ são, respectivamente, as partes real e complexa da resistividade elétrica (Dias, 2017). Determinados os valores de $\rho^{\prime}$ e $\rho^{\prime \prime}$ em (1), podemos computar os valores da amplitude da resistividade elétrica $(\Omega m)$ e a fase (mRad) para cada valor do espectro de frequências, através das seguintes expressões:

$$
\left|\rho^{*}\right|=\sqrt{\left(\rho^{\prime}\right)^{2}+\left(\rho^{\prime \prime}\right)^{2}}
$$

e

$$
\theta=\operatorname{arctg}\left(-\rho^{\prime \prime} / \rho^{\prime}\right)
$$

Obtidas as curvas de amplitude e fase da resistividade para cada amostra, realizou-se o ajuste dos dados experimentais usando dois modelos de circuitos elétricos para descrição do efeito de IP: primeiramente com o modelo de Dias e, em seguida, com um modelo composto. Os ajustes com os dois modelos foram obtidos por tentativa e erro, com qualidade verificada através de seus respectivos erros NRMSE (Normalized Root Mean Square Error). Por fim, foram realizadas estimativas da permeabilidade e raio médio de poro a partir dos parâmetros do modelo de Dias resultantes da modelagem.

\section{Modelos Elétricos para Descrição do Efeito IP: Modelo de Dias e Modelo Composto}

A função "resistividade de corrente total" do modelo de Dias para descrever a polarização elétrica em meios geológicos é dada por:

$$
\frac{\rho^{*}-\rho_{\infty}}{\rho_{0}}=\frac{m}{1+i \omega \tau^{\prime}(1+1 / \mu)},
$$

onde $i=\sqrt{-1}, \rho_{0}$ e $\rho_{\infty}$ são os valores de $\rho^{*}$ quando $f \rightarrow 0$ e $f \rightarrow \infty$, respectivamente. As funções auxiliares $\tau^{\prime}$ e $\mu$ são dadas pelas relações entre os seguintes parâmetros do modelo:

$$
\tau^{\prime}=[(1-\delta) /(1-\mathrm{m}) \delta] \tau
$$

e

$$
\mu=i \omega \tau\left[1+\eta(i \omega)^{-1 / 2}\right],
$$

onde $m$ é a cargabilidade (adimensional); $\tau$ é o tempo de relaxação da célula elementar devido à formação da estrutura da dupla camada elétrica, com $\tau<1 \mathrm{~s}$; $\delta$ é a fração unidimensional da célula elementar afetada pela polarização induzida, onde $0 \leq \delta<1 ; \quad \eta\left(\mathrm{s}^{-1 / 2}\right)$ é 0 
parâmetro eletroquímico característico (Dias, 2000; 2017).

Em Barreto \& Dias (2014), demonstrou-se que a decomposição em frações parciais da função resistividade de corrente total resulta na seguinte relação:

$$
\begin{gathered}
\frac{\rho^{*}-\rho_{\infty}}{\rho_{0}} ; \frac{m_{w}}{1+\left(i \omega \tau_{w}\right)^{1 / 2}}+\frac{m_{D}}{1+\left[\left(i \omega \tau_{D}\right)^{1 / 2}-v\right]^{2}} \\
+\frac{2 m_{D} v\left[\left(i \omega \tau_{D}\right)^{1 / 2}-v\right]}{1+\left[\left(i \omega \tau_{D}\right)^{1 / 2}-v\right]^{2}} .
\end{gathered}
$$

As definições e a inter-relação dos parâmetros derivados introduzidos pela equação (7) com os parâmetros originais do modelo de Dias vêm dadas por:

- $m_{w}$ e $m_{D}$ : são as cargabilidades parciais associadas às funções de Warburg e Debye, respectivamente, às baixas e altas frequências, e definidas pela relações: $m_{w}=m(1-\delta) /(1-m \delta)$ e $m_{D}=m-m_{w}$;

- $f_{a}$ e $f_{b}$ : fatores que medem a influência da condução ôhmica dos poros saturados livres de polarização sobre o tempo de relaxação relacionado à difusão iônica e ao efeito capacitivo, na região próxima à interface argilomineral-eletrólito, dados por: $f_{a}=\delta(1-\mathrm{m}) /(1-m \delta)$

e : $f_{b}=1-f_{a}$;

- $\tau_{w}$ e $\tau_{D}$ : tempos de relaxação relacionados, respectivamente, às baixas frequências (difusão iônica no interior da dupla camada elétrica) e às altas frequências (efeito resistivo-capacitivo da dupla camada elétrica como um todo), definidos como: $\tau_{w}=\left(f_{a} \eta\right)^{-2} \mathrm{e}$ $\tau_{D}=f_{b} \tau$

- $v$ : termo de correção de segunda ordem, dado pela relação: $v=-0,5\left(m_{w} / m\right)^{3 / 2} \eta \tau^{1 / 2}$.

A introdução do modelo composto veio da observação do registro da resistividade espectral às frequências intermediárias, onde se verificou a repetibilidade de uma possível contribuição de um efeito elétrico diferenciado em conjunto com o previsto originalmente pelo modelo de Dias para o efeito IP. Buscando aperfeiçoar o ajuste desta faixa intermediária do espectro, experimentou-se a inclusão de uma função do tipo Cole-Cole em série com a célula fundamental do modelo de Dias, que promove um efeito mais forte na fase, no intervalo intermediário das frequências, fazendo surgir um patamar de fase intermediário ou um novo máximo de fase entre o primeiro e último picos. Utilizando a versão aproximada da equação (7) (já que $v<<1$ ) e a adição de uma função de Cole-Cole, obtemos a seguinte relação para o modelo composto:

$$
\frac{\rho^{*}-\rho_{\infty}}{\rho_{0}}=\frac{m_{W 1}}{1+\left(i \omega \tau_{W 1}\right)^{1 / 2}}+\frac{m_{D}}{1+i \omega \tau_{D}}+\frac{m_{W 2}}{1+\left(i \omega \tau_{W 2}\right)^{c}},
$$

onde temos além dos parâmetros originais e derivados do modelo de Dias, a introdução de três novos: $m_{W 2}$, que é a cargabilidade parcial observada na região intermediária do espectro de frequências, que se relaciona à cargabilidade original do modelo de Dias por $m \approx m_{W 1}+m_{W 2}+m_{D} ; \quad \tau_{W 2}$ é o tempo de relaxação correspondente ao processo de difusão diferenciada, semelhante à difusão por íons, e inerente à função de Cole-Cole; $c$ é o índice empírico da função de ColeCole $(0 \leq c \leq 1)$ e característico da intensidade das heterogeneidades presentes no sistema rocha-fluido.

As heterogeneidades descritas pelo modelo composto, representada pelo surgimento de um pico ou patamar às frequências intermediárias, podem ter diferentes causas, como por exemplo: influência de características morfológicas dos argilominerais, como pore bridging, pore lining e partícula discreta; rugosidade da superfície dos grãos (Leroy et al., 2008, Revil et. al., 2014); irregularidade geométrica da superfície interna e a distribuição multimodal do tamanho dos poros da rocha.

\section{Determinação da Permeabilidade $(k)$ e Raio Médio de Poro Efetivo $\left(r_{p}\right)$}

A determinação da permeabilidade $k(\mathrm{mD})$ através de parâmetros do modelo de Dias obtidos de curvas modeladas de resistividade elétrica espectral se dá através da expressão apresentada e discutida por Barreto \& Dias (2014):

$$
k=\frac{D_{c} / \eta^{2}}{2 \xi^{2}(F-1)^{2} F \times 0.987 \times 10^{-15}},
$$

onde $D_{c}\left(\mathrm{~m}^{2} / \mathrm{s}\right)$ é o coeficiente de difusão do cátion do eletrólito, $\eta$ é o parâmetro eletroquímico que pertence ao modelo de Dias e $\xi$ é o coeficiente de cimentação e $F$ é o fator de formação da equação de Archie (1942).

A expressão para o raio de poro/garganta efetivo vem da simplificação de que um meio poroso pode ser representado por uma rede de poros capilares interconectados de raio $r_{p}$ que, segundo Marinho (2018), pode ser relacionado aos parâmetros do modelo de Dias através da seguinte expressão:

$$
r_{p}=\frac{2 f_{a} \sqrt{\tau_{W} D_{c}}}{\xi(F-1)}
$$

É interessante observar que a equação (10) pode ser reescrita com um formato similar a expressões que relacionam 0 tempo de relaxação às dimensões microgeométricas de diferentes autores na literatura, como em Tong et al. (2006) e Revil et al. (2012), através da seguinte forma similar:

$$
\tau_{W}=\left[\frac{\xi(F-1)}{2 f_{a}}\right]^{2} \frac{r_{p}^{2}}{D_{c}} .
$$




\section{Resultados e Discussões}

As amostras de rocha foram caracterizadas litologicamente como arenitos de granulação fina a média, de matriz argilosa e coloração cinza, com diferentes graus de consolidação e presença de microfissuras. Quanto à composição mineral (Tabela 1), somente os plugues de $1 \frac{1}{2}$ " puderam ser submetidos às análises por difração de raios-X, já que os plugues de 1" apresentavam baixo volume de material geológico para a pulverização requerida.

Tabela 1 - Composição Mineralógica (\%) das amostras de $11 / 2$ " de diâmetro.

\begin{tabular}{ccccc}
\cline { 2 - 5 } & $\begin{array}{c}\text { Amostra } \\
\mathbf{B C 1 - H} \\
(\mathbf{2 6 9 6 , 2 m})\end{array}$ & $\begin{array}{c}\text { Amostra } \\
\mathbf{B C 2}-\boldsymbol{H} \\
(\mathbf{2 6 9 6 , 8 m})\end{array}$ & $\begin{array}{c}\text { Amostra } \\
\mathbf{B C 3 - \boldsymbol { H }} \\
(\mathbf{2 7 0 0 , 0 )}\end{array}$ & $\begin{array}{c}\text { Amostra } \\
\mathbf{B C 4}-\boldsymbol{H} \\
(\mathbf{2 7 1 7}, \mathbf{1})\end{array}$ \\
\hline Quatzo & 11,2 & 48,9 & 49,5 & 39,0 \\
Feldspato & 41,7 & 21,7 & 29,5 & 31,4 \\
Caulinita & 8,6 & 1,4 & - & - \\
Ilita & 36,7 & 17,6 & 21,0 & 29,6 \\
Calcita & 1,8 & 10,4 & - & - \\
\hline
\end{tabular}

Quanto às medidas de petrofísica básica (Tabela 2), verificou-se que as amostras de rocha possuíam valores bons a muito bons de porosidade e permeabilidade, segundo a classificação de Levorsen (1967). No entanto, as amostras de 1" consistiam de uma zona de reservatório de qualidade inferior, devido à maior cimentação e compactação, com uma consequente rede de poros de dimensão reduzida.

Tabela 2 - Resultados das medidas de porosidade ( $\left.\phi_{\exp }\right)$ e permeabilidade ( $k_{\exp }$ ) em laboratório.

\begin{tabular}{c|cccc}
\hline $\begin{array}{c}\text { Amostras } \\
\text { de rocha }\end{array}$ & Prof. $(\boldsymbol{m})$ & Diâmetro & $\phi_{\exp }(\%)$ & $k_{\exp }(\boldsymbol{m} \boldsymbol{D})$ \\
\hline BC1-H & 2696,2 & $11 / 2 "$ & 21,7 & 23,1 \\
BC2-H & 2696,8 & $11 / 2$ & 28,6 & 82,9 \\
BC3-H & 2700,0 & $11 / 2$ & 26,8 & 30,4 \\
BC4-H & 2717,1 & $11 / 2$ & 28,1 & 44,9 \\
BC5-H & 2713,1 & $1 "$ & 19,7 & 4,4 \\
BC6-H & 2714,6 & $1 "$ & 19,0 & 7,8 \\
BC7-H & 2716,0 & $1 "$ & 19,9 & 5,5 \\
BC8-V & 2716,0 & $1 "$ & 17,0 & 4,9 \\
\hline
\end{tabular}

Apesar de as rochas apresentarem volumes porosos significativos, observa-se que os valores de $k_{\text {exp }}$ são inferiores a $100 \mathrm{mD}$. Isto está relacionado também ao significativo conteúdo de argilominerais (ilita + caulinita) preenchendo esses poros e, possivelmente, às fissuras identificadas nestes plugues, tornando estas estruturas pouco ou totalmente não-efetivas para o fluxo de fluidos.

Da etapa de modelagem das curvas de resistividade elétrica espectral, cujas curvas estão apresentadas no Apêndice $A$, verifica-se que as medidas elétricas apresentaram instabilidade nos valores da fase às baixas frequências, principalmente para as amostras de 11/2". A amostra BC1-H (45,3\% de conteúdo de argila) não apresenta um patamar acentuado e prolongado de fase nas frequências intermediárias, como observado das demais amostras de 11/2". Apesar dos registros do primeiro máximo de fase estarem pouco proeminentes dada a contribuição da condução elétrica pelos argilominerais disseminados nos poros e das poucas microfissuras efetivas (preenchidas com argilas parcialmente ou não), obteve-se para as 4 amostras de $11 \frac{1}{2}$ " bons ajustes na etapa de modelagem, como pode ser observado na Tabela 3. Utilizando o modelo de Dias, obtiveram-se erros médios de 0,028 para a amplitude e 0,480 para a fase. Já para o modelo composto, registrase um erro médio de 0,006 para a amplitude e 0,177 para a fase. Os erros menores de NRMSE para a fase ajustada com o modelo composto advém da sua boa descrição no espectro intermediário de frequências.

As curvas experimentais das amostras de 1" apresentam, em geral, patamar na fase às frequências intermediárias do espectro com intensidades de fase equivalentes às observadas nas amostras de $1 \frac{1}{2}$ ", ou até um outro máximo na fase, conforme pode ser observado nas amostras BC7-H e BC8-V. Conforme observado do espectro intermediário de frequências das curvas do Anexo $A$, há também 0 registro do efeito das heterogeneidades de cada rocha de forma similar ao verificado nas de 11/2", com forte discrepância da fase ajustada entre os dois modelos, caso em que o modelo composto produz um forte aumento no valor do tempo de relaxação $\tau_{W}$, quando comparado ao valor de ajuste usando o modelo de Dias. Apesar da impossibilidade de analisar as amostras de 1" quanto à composição mineralógica e sua influência sobre o meio geológico, verificou-se visualmente das amostras que a desagregação superficial e a presença de microfissuras podem ter contribuído para as instabilidades observadas, assim como a não formação de um patamar assintótico às baixas frequências. Mesmo com essas considerações, foram realizados bons ajustes para as curvas dessas amostras, com erros médios de 0,058 para a amplitude e 0,488 para a fase, ajustas pelo modelo de Dias, e 0,031 para a amplitude e 0,189 para a fase, ajustadas pelo modelo composto.

Tabela 3 - Erros NRMSE para as amostras de rocha obtidas da aplicação dos dois modelos elétricos

\begin{tabular}{c|cc||cc}
\cline { 2 - 3 } & \multicolumn{2}{c||}{ Modelo de Dias } & \multicolumn{2}{c}{ Modelo Composto } \\
\cline { 2 - 5 } & Amplitude & Fase & Amplitude & Fase \\
\hline BC1-H & 0,013 & 0,398 & 0,010 & 0,345 \\
BC2-H & 0,021 & 0,482 & 0,003 & 0,177 \\
BC3-H & 0,029 & 0,461 & 0,006 & 0,110 \\
BC4-H & 0,050 & 0,579 & 0,006 & 0,076 \\
BC5-H & 0,027 & 0,285 & 0,034 & 0,255 \\
BC6-H & 0,034 & 0,490 & 0,004 & 0,146 \\
BC7-H & 0,121 & 0,699 & 0,067 & 0,137 \\
BC8-V & 0,052 & 0,478 & 0,018 & 0,218 \\
\hline
\end{tabular}


Observa-se ainda dos dados uma correlação entre a instabilidade verificada na medida da fase (às baixas frequências) e o surgimento do patamar de fase (às frequências intermediárias do espectro) atribuído a heterogeneidades das amostras. Nas baixas frequências, a medida consumindo um tempo mais longo, estariam sendo perturbada por um processo de difusão diferenciado ocorrendo simultaneamente com a medida, através das microfissuras efetivas ou parcialmente efetivas. Nas frequências intermediárias, o tempo de relaxação $\tau_{W 2}$, de valor entre $\tau_{W 1}$ e $\tau_{D}$, estaria mostrando a presença de um processo de difusão "imperfeito" de íons da solução, em menor profundidade do que aquela associada a $\tau_{W 1}$. É possível que esse fenômeno tenha relação com a superfície interna irregular dos poros promovida pelas características texturais da ilita (pore-bridging), argilomineral presente em maior volume em todas as amostras submetidas à análise mineralógica, e que figura como o principal causador do efeito IP. Estruturas de pore-bridging tem a tendência a interligar as paredes da rede de poros, favorecendo que a zona polarizada em formação em uma lateral do poro "interaja" com outra zona polarizada próxima durante o processo de difusão de íons. Por outro lado, uma rede de poros diversificada em termos de volume, com superfície e geometria irregulares, associada a argilomineraiss dispersos em seu interior, poderia influenciar o efeito de difusão. Os valores mais baixos do parâmetro de ColeCole $(c<0,5)$ também vem associados aos patamares mais longos nas frequências intermediárias e, provavelmente, às ocorrências mais acentuadas ou distribuídas das heterogeneidades citadas no interior das amostras de rocha analisadas.

A amostra $\mathrm{BC} 5-\mathrm{H}$ apresenta um único e intenso $1^{\circ}$ máximo de fase associado à difusão, mas também um forte declínio da amplitude da resistividade no mesmo intervalo de frequências $(1 \mathrm{mHz}$ a $1 \mathrm{~Hz})$, em que se situa o máximo da fase. Ao exibir um único máximo, mostra que o efeito devido à corrente de indução é insignificante, nesta amostra. É importante observar que, em geral, o $1^{\circ}$ máximo vem bem descrito por ambos os modelos utilizados. Das curvas experimentais das amostras BC7$\mathrm{H}$ e BC8-V, por serem provenientes da mesma profundidade, chama a atenção 0 registro de características de anisotropia da resistividade elétrica, como: (a) o valor da resistividade DC na horizontal ser cerca de 8 vezes o seu valor na vertical, sendo BC8-V menos porosa e permeável, conforme observado da Tabela 2. O forte efeito de difusão na amostra BC7-H parece exercer uma espécie de bloqueio à circulação da corrente de indução; (b) o processo de difusão é mais intenso na horizontal do que na vertical, inclusive observando-se um $2^{\circ}$ máximo de fase proeminente na horizontal (muito próximo do 1ำ máximo), associado provavelmente com um segundo processo de difusão relacionado com a densidade de heterogeneidades, sem um correspondente na vertical de igual intensidade; (c) o efeito resistivo-capacitivo na vertical é muito intenso, provavelmente em decorrência da estratificação, sendo quase inexistente na horizontal.
Estimativas de Permeabilidade e Raio Médio de Poro Efetivo das Amostras de Rocha

Utilizando a equação (9) com $D_{c}$ igual o coeficiente de difusão do sódio $\left(1,3 \times 10^{-9} \mathrm{~m}^{2} / \mathrm{s}\right.$, segundo Bockris \& Reddy (2000)) e os parâmetros do modelo de Dias obtidos da modelagem dos dados experimentais (Apêndice A), foram produzidas estimativas para a permeabilidade $k(\mathrm{mD})$ com valores teóricos muito próximos para a permeabilidade medida em laboratório, conforme pode ser observado na Figura 2. Isso foi possível pois ambos os modelos descreveram com boa precisão e de forma quase igual o primeiro máximo relacionado ao efeito de difusão. Destaca-se que o ajuste de menor qualidade promovido pelo modelo de Dias no restante do espectro não interferiu na qualidade dessas estimativas.

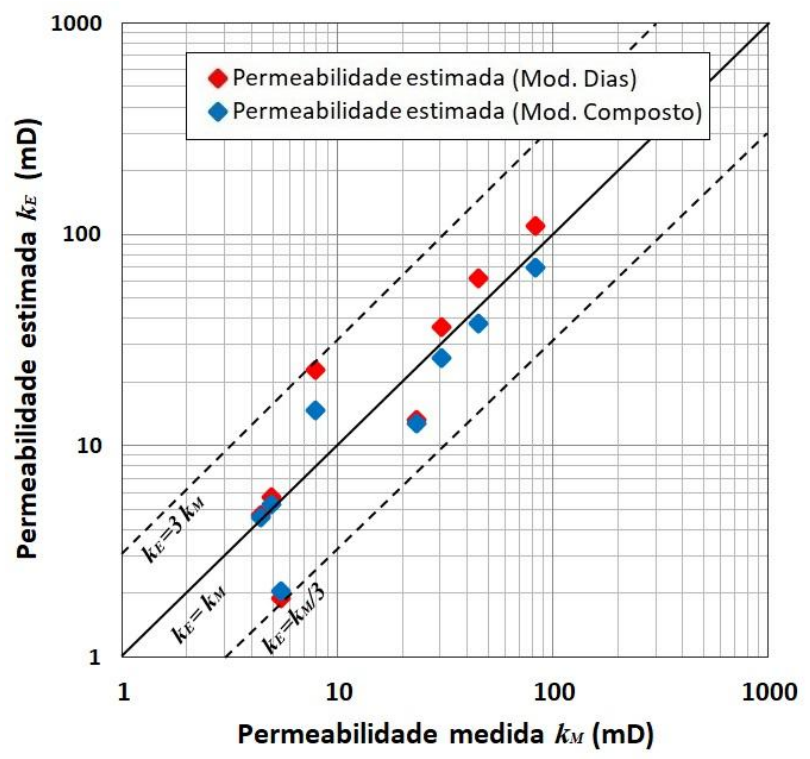

Figura 2 - Gráfico ilustrativo da correlação entre os valores medidos e os valores estimados da permeabilidade das amostras de rocha.

Podemos verificar quantitativamente a qualidade das estimativas de permeabilidade realizadas através da aplicação do erro residual logarítmico mínimo quadrado $(R)$, dado pela expressão:

$$
[\ln (R)]^{2}=\frac{1}{N} \sum_{j=1}^{N}\left[\ln k_{M}(j)-\ln k_{E}(j)\right]^{2},
$$

onde $k_{M}$ e $k_{E}$ são, respectivamente, os valores de permeabilidade medidos e estimados; e $N$ o número total de medidas consideradas. Da aplicação da equação (12), obteve-se os seguintes valores de $R: 1,27$ para o modelo original de Dias e 1,16 para o modelo composto. Faz-se destaque que ambos os valores citados de $R$ são inferiores ao menor erro deste tipo registrado na lituratura, pertencente ao trabalho de Tong et al. (2006): $R=1,76$. 
Utilizando a equação (10) para o raio médio de poro efetivo $\left(r_{p}\right)$ e os parâmetros obtidos dos ajustes promovidos pelos modelos de Dias e o modelo composto, foi possível estimar valores de $r_{p}$, em $\mu \mathrm{m}$, para cada espécimen de rocha. No entanto, não foi possível realizar uma análise comparativa destas estimativas com dados experimentais de raio ou garganta de poro, pois 0 laboratório de petrofísica do LENEP não possui os equipamentos requeridos para a realização dos experimentos. Buscou-se, então, um meio alternativo para estimar este parâmetro através do método proposto por Pittman (1992), muito usado na indústria petroleira atualmente, utilizando suas equações empíricas para o cálculo do raio de poro denominadas $r 10$ e r50. Observase da Tabela 4, que os dados estimados de raio médio de poro efetivo pela equação (10) apresentam correspondência de $100 \%$ quando comparados ao intervalo obtido pelo método de Pittman, para ambos os modelos.

Tabela 4 - Valores estimados de raio médio de poro efetivo $r_{p}$ usando parâmetros obtidos do modelo de Dias e do modelo composto comparados ao intervalo de raio de poro estimados usando o método de Pittman.

\begin{tabular}{|c|c|c|c|c|}
\hline Amostras & $\begin{array}{c}\boldsymbol{r}_{p-\text { Dias }} \\
(\mu \mathrm{m})\end{array}$ & $\begin{array}{c}\boldsymbol{r}_{p-\text { Comp }} \\
(\mu \mathrm{m})\end{array}$ & $\begin{array}{c}\text { Intervalo - } \\
\text { Pittman } \\
(\mu \mathrm{m}) \\
\end{array}$ & $\begin{array}{c}\text { Dentro } \\
\text { do } \\
\text { intervalo }\end{array}$ \\
\hline BC1-H & 1,5 & 1,5 & $1,0-4,2$ & Sim \\
\hline BC2-H & 3,2 & 2,6 & $1,7-7,2$ & Sim \\
\hline ВC3-H & 2,0 & 1,7 & $1,0-4,5$ & Sim \\
\hline BC4-H & 2,5 & 2,0 & $1,2-5,3$ & Sim \\
\hline BC5-H & 1,0 & 1,0 & $0,4-1,9$ & Sim \\
\hline BC6-H & 1,8 & 1,6 & $0,6-2,6$ & Sim \\
\hline BC7-H & 0,6 & 0,6 & $0,5-2,1$ & Sim \\
\hline BC8-V & 1,2 & 1,2 & $0,5-2,1$ & Sim \\
\hline
\end{tabular}

\section{Conclusões}

Foi possível obter bons registros do Efeito IP das amostras de rocha da Bacia de Campos. Os dados de resistividade elétrica medidos em laboratório apresentam com regularidade um patamar de fase às frequências intermediárias, colocando dificuldade ao modelo de Dias para ajustar os dados neste intervalo do espectro. No entanto, com a introdução de um modelo composto para melhor ajustar estes dados, o problema foi contornado, sem haver comprometimento da boa descrição dos máximos de fase originalmente previsto pelo modelo de Dias. Acreditamos que as heterogeneidades comentadas neste trabalho (características texturais das argilas, geometria e distribuição de poro complexa) possam ser as causadoras deste fenômeno às frequências intermediárias, onde o modelo composto complementa satisfatoriamente o ajuste de dados.

Quanto aos valores finais de permeabilidade e raio de poro efetivos estimados utilizando parâmetros dos dois modelos, foram obtidos resultados muito próximos dos valores experimentais de $\mathrm{k}$ e dentro dos intervalos de raios de poro determinados usando o método de Pittman. Isto evidencia a importância da boa descrição do primeiro máximo de fase, às baixas frequências, precisamente entre $10 \mathrm{mHz}$ a $100 \mathrm{~Hz}$, sendo esse intervalo de crucial importância para p planejamento e realização de medidas in situ das propriedades de reservatório discutidas.

\section{Agradecimentos}

Os autores agradecem ao LENEP - Laboratório de Engenharia e Exploração de Petróleo da UENF pela excelente infraestrutura disponibilizada em seu laboratório de petrofísica. Agradecemos à ANP/ PETROBRAS por fornecer as amostras de rocha aqui analisadas. Agradecemos também à PETROBRAS pelo financiamento desta pesquisa.

\section{Referências}

API, 1998. RP40 - Recommended practices for core analysis. $2^{\text {nd }}$ ed., API (American Petroleum Institute) Publishing Series, Washington (DC), USA.

Archie, G. E., 1942. The electrical resistivity log as an aid in determining some reservoir characteristics, Journal of Petroleum Technology, 1, 55-62.

Barreto, A. N. \& Dias, C. A., 2014. Fluid salinity, clay content and permeability of rocks determined through complex resistivity partition fraction decomposition. Geophysics, 79 (5), D333-D347.

Bear, J., 1972. Dynamics of Fluids in Porous Media. $1^{\text {st }}$ ed., American Elsevier Publishing Company, Inc., New York, USA.

Bockris, J. O. \& Reddy, A. K. N., 2000. Modern electrochemistry, $2^{\text {nd }}$ Ed., Kluwer Academic Publishers, New York, USA, vol. 1.

Dias, C. A., 1968. A non-grounded method for measuring electrical induced polarization and conductivity: Ph.D. thesis, University of California-Berkeley.

Dias, C.A., 1972. An analytical model for a polarizable medium at radio and lower frequencies, J. Geophys. Res., 77, 4945-4956.

Dias, C. A., 2000. Developments in a model to describe low-frequency electrical polarization of rocks. Geophysics, $65,2,437-451$.

Dias, C. A., 2017. Método geofísico eletromagnético a multifrequência: um invento brasileiro. SBGf, Blue Print Gráfica e Editora, Rio de Janeiro, Brasil.

Leroy, P., Revil, A., Kemna, A., Cosenza, P. \& Ghorbani, A., 2008. Complex conductivity of water-saturated packs of glass beads. Journal of Colloid and Interface Science, Elsevier BV, v. 321, n. 1, p. 103-117. 
Levorsen, A. I., 1967. Geology of petroleum. 2nd Ed.. WH Freeman, San Francisco, USA.

Maerefat, N., Baldwin, B., Chaves, A., Latorranca, G., Swanson, B. et al., 1990. SCA guidelines for sample preparation and porosity measurement of electrical resistivity samples. part IV - guidelines for saturating and desaturating core plugs during electrical resistivity measurements. The Log Analyst, Society of Petrophysicists and Well-Log Analysts, 02, v. 31.

Marinho, 2018. Estudo do efeito IP observado em amostras de rocha do Campo de Corvina, Bacia de Campos, através de modelos elétricos espectrais. Dissertação de Mestrado, UENF, Macaé, Brasil.

Pelton, W. H.; Ward, S. H.; Hallof, P. G.; Sill, W. R. \& Nelson, P. H, 1978. Mineral discrimination and removal of inductive coupling with multi-frequency IP. Geophysics, Society of Exploration Geophysicists, v. 43, n. 3, p. 588609.

Pittman, E. D., 1992. Relationship of porosity and permeability to various parameters derived from mercury injection-capillary pressure curves for sandstone (1). AAPG Bulletin, v. 76, n. 2.

Revil, A. \& Florsch, N., 2010. Determination of permeability from spectral induced polarization in granular media. Geophysical Journal International, 181, 14801498.

Revil, A., Florsch, N. \& Carmerlynck, C., 2014. Spectral induced polarization porosimetry. Geophysical Journal International, 198, 1016-1033.

Revil, A., Koch, K. \& Hollinger, K., 2012. Is it the grain size or the characteristic pore size that controls the induced polarization relaxation time of clean sands and sandstones? Water Resources Research, 48, W05602.

Rietveld, H. M. A, 1969. Profile refinement method for nuclear and magnetic structures. Journal of Applied Crystallography, International Union of Crystallography (IUCr), 2, 2, 65-71.

Tong, M., Li, L., Wang, W., \& Jiang, Y., 2006. A time domain induced-polarization method for estimating permeability in a shaly sand reservoir. Geophys. Prosp., $54,623-631$.

\section{Apêndice A - Medidas de Resistividade Complexa Espectral e suas Modelagens}
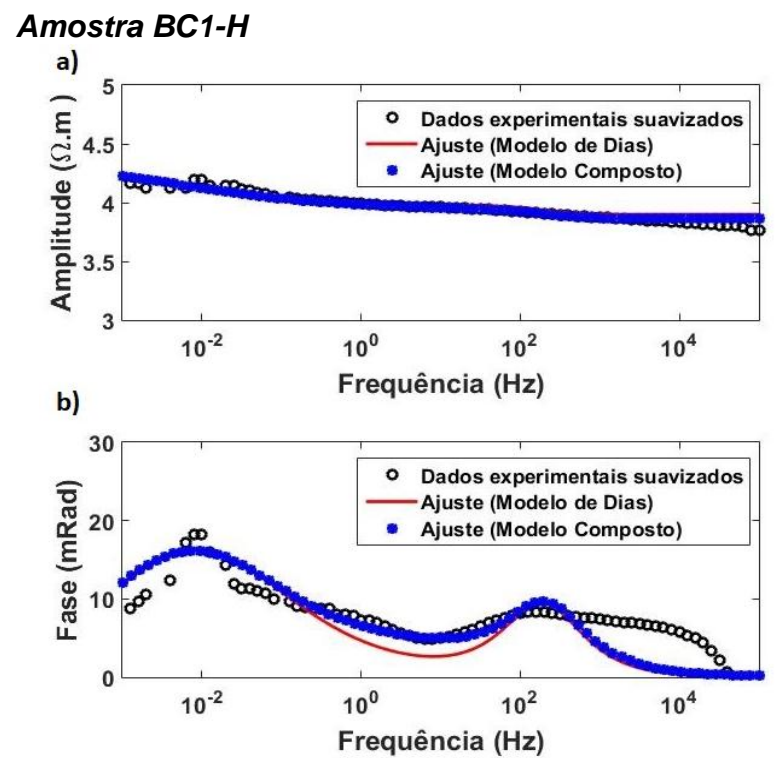

\begin{tabular}{l|lc}
\hline \multicolumn{1}{c|}{ Modelo de Dias } & \multicolumn{2}{|c}{ Modelo Composto } \\
\hline$\rho_{0}=4,3 \Omega \mathrm{m} ; \quad m=0,093 ;$ & $\rho_{0}=4,3 \Omega \mathrm{m} ;$ & $m=0,102 ;$ \\
$\tau=1,0 \times 10^{-3} s ; \quad \delta=0,20 ;$ & $\tau=9,0 \times 10^{-4} \mathrm{~s} ;$ & $\delta=0,18 ;$ \\
$\eta=1,21 \mathrm{~s}^{-1 / 2}$ & $\eta=1,23 \mathrm{~s}^{-1 / 2} ;$ & $m_{w 2}=0,015 ;$ \\
& $\tau_{w 2}=3,2 \times 10^{-2} \mathrm{~s} ; \quad \mathrm{c}=0,42$ \\
\hline
\end{tabular}

\section{Amostra BC2-H}

a)

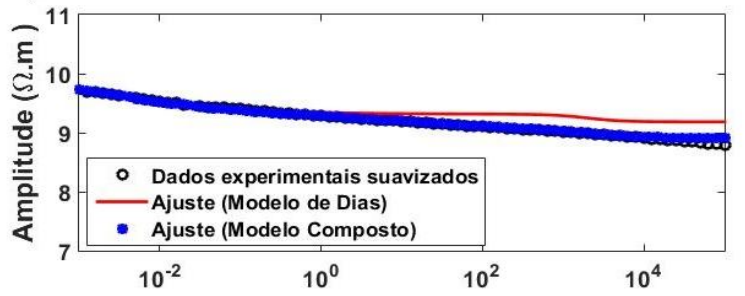

b) Frequência $(\mathrm{Hz})$

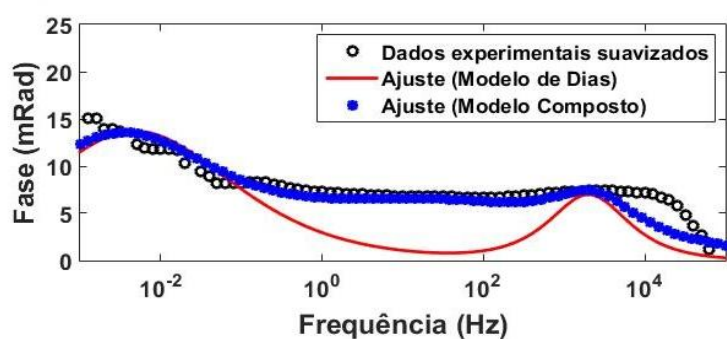

\begin{tabular}{l|lc}
\hline \multicolumn{2}{c|}{ Modelo de Dias } & \multicolumn{2}{c}{ Modelo Composto } \\
\hline$\rho_{0}=9,95 \Omega \mathrm{m} ; \quad m=0,077 ;$ & $\rho_{0}=9,95 \Omega \mathrm{m} ;$ & $m=0,108 ;$ \\
$\tau=9,5 \times 10^{-5} \mathrm{~s} ; \quad \delta=0,18 ;$ & $\tau=7,5 \times 10^{-5} \mathrm{~s} ; \quad \delta=0,11 ;$ \\
$\eta=1,00 \mathrm{~s}^{-1 / 2}$ & $\eta=1,25 \mathrm{~s}^{-1 / 2} ; \quad m_{w 2}=0,043 ;$ \\
& $\tau_{w 2}=6,4 \times 10^{-3} \mathrm{~s} ; \quad \mathrm{c}=0,32$
\end{tabular}




\section{Amostra BC3-H}

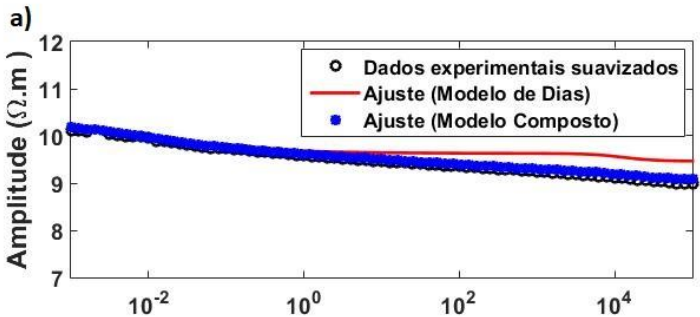

b)

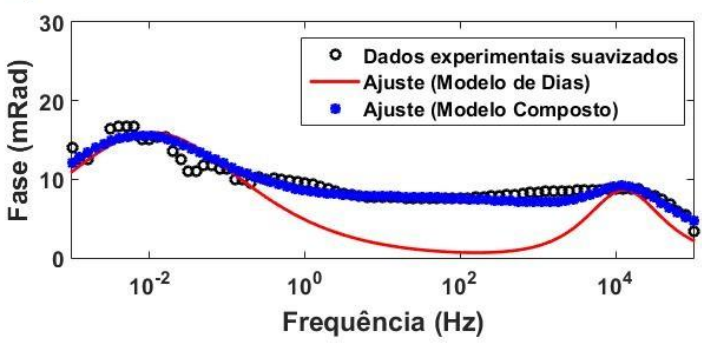

\begin{tabular}{l|lc}
\hline \multicolumn{1}{c|}{ Modelo de Dias } & \multicolumn{2}{c}{ Modelo Composto } \\
\hline$\rho_{0}=10,4 \Omega \mathrm{m} ; \quad m=0,090 ;$ & $\rho_{0}=10,4 \Omega \mathrm{m} ;$ & $m=0,135 ;$ \\
$\tau=1,5 \times 10^{-5} \mathrm{~s} ; \quad \delta=0,19 ;$ & $\tau=1,2 \times 10^{-5} \mathrm{~s} ;$ & $\delta=0,12 ;$ \\
$\eta=1,41 s^{-1 / 2}$ & $\eta=1,67 \mathrm{~s}^{-1 / 2} ; \quad m_{w 2}=0,065 ;$ \\
& $\tau_{w 2}=2,6 \times 10^{-3} \mathrm{~s} ; \quad \mathrm{c}=0,25$
\end{tabular}

\section{Amostra BC4-H}

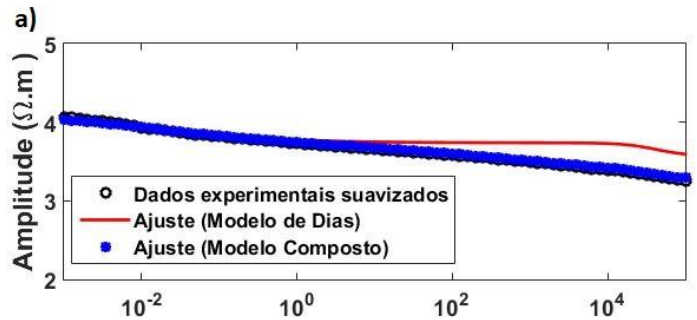

b)

Frequência $(\mathrm{Hz})$

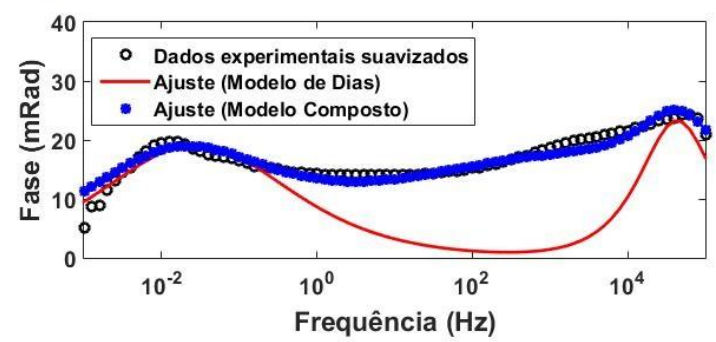

\begin{tabular}{l|lc}
\hline \multicolumn{1}{c|}{ Modelo de Dias } & \multicolumn{2}{c}{ Modelo Composto } \\
\hline$\rho_{0}=4,1 \Omega \mathrm{m} ; \quad m=0,130 ;$ & $\rho_{0}=4,1 \Omega \mathrm{m} ;$ & $m=0,240 ;$ \\
$\tau=5,5 \times 10^{-6} s ; \quad \delta=0,35 ;$ & $\tau=4,3 \times 10^{-6} \mathrm{~s} ;$ & $\delta=0,20 ;$ \\
$\eta=1,25 s^{-1 / 2}$ & $\eta=1,60 \mathrm{~s}^{-1 / 2} ; \quad m_{w 2}=0,152 ;$ \\
& $\tau_{w 2}=1,1 \times 10^{-4} \mathrm{~s} ;$ & $\mathrm{c}=0,24$ \\
\hline
\end{tabular}

\section{Amostra BC5-H}

a)

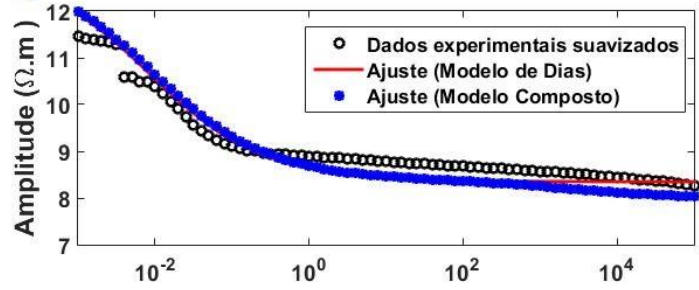

Frequência (Hz)

b)

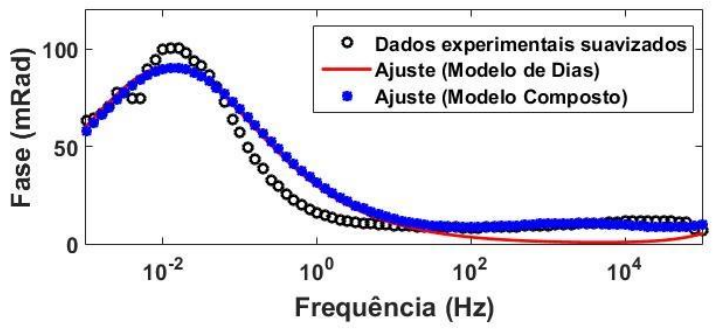

\begin{tabular}{l|lc}
\hline \multicolumn{2}{c|}{ Modelo de Dias } & \multicolumn{2}{c}{ Modelo Composto } \\
\hline$\rho_{0}=13,0 \Omega \mathrm{m} ; \quad m=0,420 ;$ & $\rho_{0}=13,0 \Omega \mathrm{m} ;$ & $m=0,450 ;$ \\
$\tau=1,0 \times 10^{-7} s ; \quad \delta=0,23 ;$ & $\tau=1,0 \times 10^{-7} \mathrm{~s} ;$ & $\delta=0,24 ;$ \\
$\eta=1,50 \mathrm{~s}^{-1 / 2}$ & $\eta=1,52 \mathrm{~s}^{-1 / 2} ; \quad m_{w 2}=0,030 ;$ \\
& $\tau_{w 2}=6,4 \times 10^{-5} \mathrm{~s} ; \quad \mathrm{c}=0,50$
\end{tabular}

\section{Amostra BC6-H}

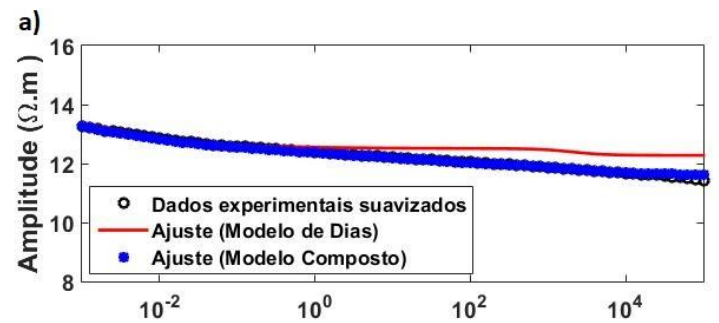

b) Frequência $(\mathrm{Hz})$

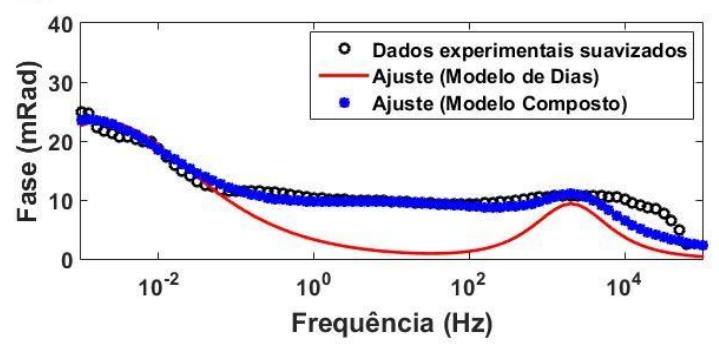

\begin{tabular}{l|lc}
\hline \multicolumn{1}{c|}{ Modelo de Dias } & \multicolumn{2}{c}{ Modelo Composto } \\
\hline$\rho_{0}=14,0 \Omega \mathrm{m} ; \quad m=0,123 ;$ & $\rho_{0}=14,0 \Omega \mathrm{m} ;$ & $m=0,173 ;$ \\
$\tau=9,0 \times 10^{-5} s ; \quad \delta=0,15 ;$ & $\tau=7,0 \times 10^{-5} \mathrm{~s} ;$ & $\delta=0,10 ;$ \\
$\eta=0,77 \mathrm{~s}^{-1 / 2}$ & $\eta=0,87 \mathrm{~s}^{-1 / 2} ;$ & $m_{w 2}=0,062 ;$ \\
& $\tau_{w 2}=1,1 \times 10^{-2} \mathrm{~s} ;$ & $\mathrm{c}=0,31$
\end{tabular}




\section{Amostra BC7-H}

a)

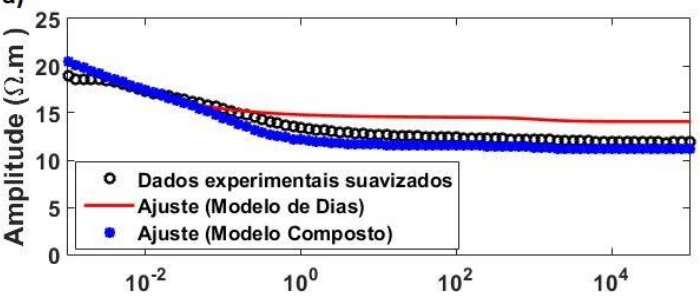

b)

Frequência $(\mathrm{Hz})$

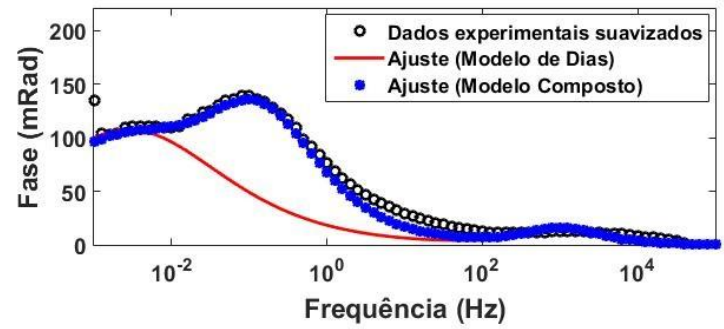

Modelo de Dias $\quad$ Modelo Composto

\begin{tabular}{ll|lc}
$\rho_{0}=24,5 \Omega \mathrm{m} ;$ & $m=0,425 ;$ & $\rho_{0}=24,5 \Omega \mathrm{m} ;$ & $m=0,545 ;$ \\
$\tau=1,4 \times 10^{-4} s ; \quad \delta=0,07 ;$ & $\tau=1,5 \times 10^{-4} \mathrm{~s} ;$ & $\delta=0,06 ;$ \\
$\eta=2,45 \mathrm{~s}^{-1 / 2}$ & $\eta=2,35 \mathrm{~s}^{-1 / 2} ;$ & $m_{w 2}=0,165 ;$ \\
& $\tau_{w 2}=1,45 \mathrm{~s} ;$ & $\mathrm{c}=0,75$
\end{tabular}

\section{Amostra BC8-V}

a)

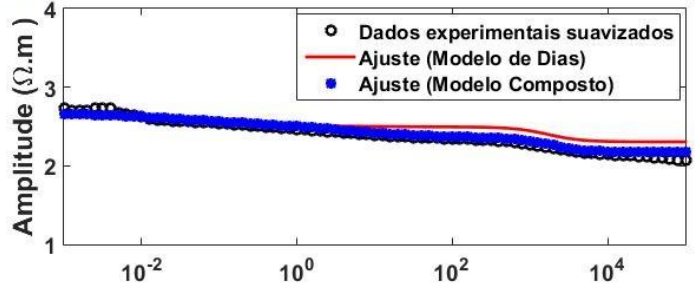

b)

Frequência $(\mathrm{Hz})$

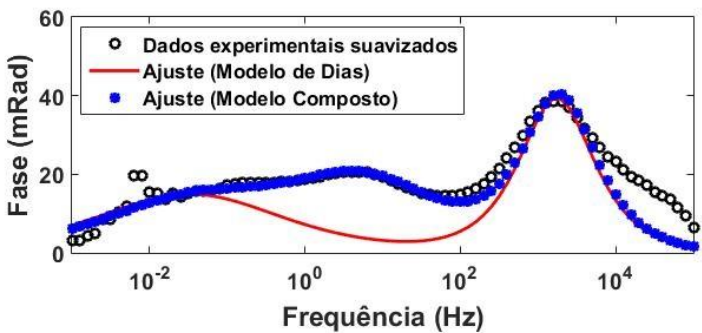

Modelo de Dias $\quad$ Modelo Composto

$\rho_{0}=2,68 \Omega \mathrm{m} ; \quad m=0,140 ; \quad \rho_{0}=2,68 \Omega \mathrm{m} ; \quad m=0,188 ;$

$\tau=1,9 \times 10^{-4} s ; \quad \delta=0,54 ; \quad \tau=1,8 \times 10^{-4} \mathrm{~s} ; \quad \delta=0,55 ;$

$\eta=0,87 \mathrm{~s}^{-1 / 2} \quad \eta=0,90 \mathrm{~s}^{-1 / 2} ; \quad m_{w 2}=0,061 ;$

$\tau_{w 2}=2,9 \times 10^{-2} \mathrm{~s} ; \quad \mathrm{c}=0,60$ 